\title{
Mother-to-Child Transmission of HIV Infection and Its Determinants among Exposed Infants on Care and Follow-Up in Dire Dawa City, Eastern Ethiopia
}

\author{
Fisseha Wudineh ${ }^{1}$ and Bereket Damtew ${ }^{2}$ \\ ${ }^{1}$ Pediatric ART Clinic, ART Department, Dil Chora Referral Hospital, Dire Dawa, Ethiopia \\ ${ }^{2}$ Department of Epidemiology and Biostatistics, College of Health and Medical Sciences, Haramaya University, Harar, Ethiopia
}

Correspondence should be addressed to Bereket Damtew; bekiofdu@gmail.com

Received 10 November 2015; Revised 16 January 2016; Accepted 18 January 2016

Academic Editor: Soraya Seedat

Copyright (c) 2016 F. Wudineh and B. Damtew. This is an open access article distributed under the Creative Commons Attribution License, which permits unrestricted use, distribution, and reproduction in any medium, provided the original work is properly cited.

\begin{abstract}
Since the scale-up for prevention of mother-to-child transmission (PMTCT) services, rates of HIV infection among exposed infants have significantly declined. However, current achievements fell short of achieving the target sets. We investigated mother-to-child transmission (MTCT) of HIV infection and its determinants among HIV-exposed infants on care at Dilchora Referral Hospital in Dire Dawa City Administration. A retrospective institutional cohort study was conducted by reviewing follow-up records of HIV-exposed infants who were enrolled into care. Infants' HIV serostatus was the outcome measure of the study. Bivariate and multivariate logistic regressions were employed to identify significant determinants. Of the $382 \mathrm{HIV}$-exposed infants enrolled into care, 60 (15.7\%) became HIV positive. Rural residence (AOR: 3.29; 95\% CI: 1.40, 7.22), home delivery (AOR: 3.35; 95\% CI: 1.58, 8.38), infant not receiving ARV prophylaxis at birth (AOR: 5.83; 95\% CI: 2.84, 11.94), mixed feeding practices (AOR: 42.21; 95\% CI: 8.31, 214.38), and mother-child pairs neither receiving ARV (AOR: 4.42; 95\% CI: 2.01, 9.82) were significant independent determinants of MTCT of HIV infection. Our findings suggest additional efforts to intensify scale-up of PMTCT services in rural setting and improve institutional delivery and postnatal care for HIV positive mothers and proper follow-up for HIV-exposed infants.
\end{abstract}

\section{Introduction}

In 2011, there were approximately 330,000 children newly infected with Human Immunodeficiency Virus (HIV); most of the infections being occurring in sub-Saharan African countries [1]. Ethiopia is among the ten countries in the world with the highest burden of HIV infections among children where mother-to-child transmission (MTCT) occurs in a third of infants born to HIV-infected mothers. In 2012, HIV prevalence at antenatal care clinics was $2.4 \%$ and MTCT ranging from 17\% (at 6 weeks) to 30\% (including breastfeeding), amounting to an estimated 13,000 new HIV infections among children $[1,2]$.

Since the Ethiopian Ministry of Health launched the national program for prevention of mother-to-child transmission (PMTCT) of HIV infection in 2001, the number of facilities with PMTCT service has reached 1,445 providing
ARV prophylaxis for 10,302 HIV positive pregnant women and 4,945 exposed infants in 2011 [3]. Although prophylactic medication coverage during pregnancy has improved significantly in east and southern Africa, it is still lower than the $80 \%$ target due to limited healthcare provision during childbirth [1]. In Ethiopia, while only $40 \%$ of identified HIV positive pregnant women received ARV prophylaxis (24\% of the estimated 42,900 in need), less than a quarter of newborns to HIV positive women received ARV prophylaxis and only 4,753 (11\%) exposed infants received virological test for HIV within 6 weeks of birth [4]. Low coverage of PMTCT services has been a consistent problem as nearly $82 \%$ of women accessed ANC services at least once during their most recent pregnancy; PMTCT services were available only in $54 \%$ of all facilities. Furthermore, while $98 \%$ of pregnant women attending ANC clinics providing PMTCT services were counseled, a quarter were not tested for HIV, and even among those who 
were HIV positive, $60 \%$ were not provided with ARV prophylaxis for PMTCT $[3,4]$.

In resource-limited countries with high prevalence of MTCT of HIV infection, where PMTCT is not widespread and early infant diagnosis (EID) is still a challenge, assessing the risk factors for MTCT will help to decrease child morbidity and mortality and strengthen PMTCT programs [5]. With this regard, few studies have described the magnitude of MTCT and assess efforts made towards PMTCT [6-8]; there is dearth of evidence regarding factors determining MTCT HIV infection to infants born to HIV positive mothers. Therefore, this study determines prevalence of mother-tochild transmission of HIV infection and its determinants among exposed infants in care at Dire Dawa Referral Hospital.

\section{Methods}

2.1. Study Setting. This study was conducted at Dil Chora Referral Hospital, in Dire Dawa City Administration. Dire Dawa is located $515 \mathrm{Km}$ to the east of the capital city, Addis Ababa. The city has one referral hospital; four private hospitals; fifteen ( 9 in the city and 6 in rural kebeles) health centers; four private higher clinics; and one specialized STI and HIV care clinic. The referral hospital provides comprehensive HIV treatment and care as well as PMTCT service. At the time of the study there were 419 infants at the HIV-exposed infants clinic.

2.2. Study Design. Retrospective institution based follow-up study design was used to investigate factors associated with HIV transmission among infants born to HIV positive mothers who were on follow-up at the HIV-exposed infant care clinic. A total of $382 \mathrm{HIV}$-exposed infants who received care and support between July 2005 and July 2013 at the exposed infant follow-up clinic were included in the study. The independent variables were maternal factors (such as on HAART or prophylaxis, place of delivery) and infant factors (such as feeding options, follow-up). The study's outcome measure was HIV serostatus at the end of the follow-up.

2.3. Participants. The source population was all infants on follow-up in the HIV-exposed infant care clinic at Dil Chora Referral Hospital. The study population was comprised of exposed infants who were on follow-up care between July 2005 and July 2013 at the clinic and for which a confirmatory HIV test was done. Exposed infants $<6$ weeks of age, lost to follow-up, transferred out, or dead without confirmatory test were excluded from the study. Minimum sample size determination was made by considering a vertical HIV transmission rate of $10 \%$ among exposed infants on care from a previous study [7]. Study participants were selected by simple random sampling using their unique National PMTCT identification number.

2.4. Data Collection and Quality Control. A structured data collection tool was developed to compile the required information by adopting the national HIV-exposed infant followup form. The data were collected by reviewing mothers'
PMTCT and exposed infants' care follow-up records at the antenatal care (ANC) clinic and exposed infant follow-up clinics, respectively.

The data were collected by two experienced nurses (one from ANC and one from exposed infant clinic) trained on comprehensive HIV care, PMTCT, and exposed infant care and who are working at the respective clinics in Dil Chora Referral Hospital. The data collectors were trained on the data collection procedures for one day. A pretest of the data collection tool and data collection process was made at Sabiyan Health Center found in the city. The investigator oversaw the overall process. All completed data collection forms were examined for completeness, consistency, and clarity during data management, storage, and analysis.

2.5. Follow-Up and Measurements. According to the national HIV-exposed infant care guideline, HIV diagnosis of exposed infants is made by a positive virological test using Deoxyribonucleic Acid-Polymerase Chain Reaction (DNA-PCR) test at 6 weeks or as early as possible thereafter, or if she/he displays any severe classifications possibly due to HIV or has positive antibody test under 18 months and 2 or more of the following: oral thrush, severe pneumonia, or severe sepsis. The child will be referred to ART care clinic after positive HIV diagnosis [4]. ARV prophylaxis follow-up schedule of infants born to HIV positive mothers is at 6 hours after birth, at 6 th day, and then at the 6 th, 10 th, and 14 th week of life. Thereafter, it is on monthly basis until 6th month of age and every 3 months until age of 18 months for asymptomatic infants. Cotrimoxazole prophylaxis is usually started when the infant is aged $4-6$ weeks $[3,4]$. However, children were followed up for variable periods of time as their HIV infection serostatus may be declared at different times.

2.6. Data Analysis. Exploration of data was made to check for any inconsistencies, coding error, out of range, and missing values and appropriate corrections were made. Descriptive analyses of sociodemographic information, infant prophylaxis, and infant follow-up information, maternal PMTCT interventions, and infant final HIV serostatus were carried out. Bivariate logistic regression model was used to assess associations of independent variables with the outcome variable and calculate their crude odds ratios. Finally, all variables significant at $P \leq 0.2$ in the bivariate model were included in the final multivariate logistic regression model and their adjusted odds ratios were calculated. Variables significant at $P<0.05$ in the final model were considered as independent determinant factors for infant HIV serostatus. And crosstabs were done to show the rate of each variable with the outcome. All analyses were conducted using SPSS version 16.0 for Windows (SPSS ${ }^{\circledast}$ Inc., Chicago, IL, USA).

\section{Results}

A total of $382 \mathrm{HIV}$-exposed infants on care were included in the study. One hundred ninety-three $(50.5 \%)$ were females and majority 341 (89.3\%) were from urban areas. Regarding place of delivery, 344 (90.1\%) of the HIV-exposed infants were delivered at a health institution. 
TABLE 1: Sociodemographic and clinical characteristics of HIV-exposed infants on follow-up care in Dire Dawa, Ethiopia, September 2014.

\begin{tabular}{|c|c|c|c|}
\hline Variables & Total, N (\%) & Negative, $N(\%)$ & Positive, $N(\%)$ \\
\hline \multicolumn{4}{|l|}{ Sex of infant } \\
\hline Male & $189(49.5 \%)$ & $163(86.2 \%)$ & $26(13.8 \%)$ \\
\hline Female & $193(50.5 \%)$ & $159(82.4 \%)$ & $34(17.6 \%)$ \\
\hline \multicolumn{4}{|l|}{ Infant age at enrollment } \\
\hline$\leq 6$ weeks & $319(83.5 \%)$ & $275(86.2 \%)$ & $44(13.8 \%)$ \\
\hline$>6$ weeks & $63(16.5 \%)$ & $47(74.6 \%)$ & $16(25.4 \%)$ \\
\hline \multicolumn{4}{|l|}{ Residence } \\
\hline Urban & $341(89.3 \%)$ & $296(86.8 \%)$ & $45(13.2 \%)$ \\
\hline Rural & $41(10.7 \%)$ & $26(63.4 \%)$ & $15(36.6 \%)$ \\
\hline \multicolumn{4}{|l|}{ Place of delivery } \\
\hline Health institution & $344(90.1 \%)$ & $303(88.1 \%)$ & $41(11.9 \%)$ \\
\hline Home & $38(9.9 \%)$ & $19(50.0 \%)$ & $19(50.0 \%)$ \\
\hline \multicolumn{4}{|l|}{ PMTCT intervention } \\
\hline On ART & $100(26.2 \%)$ & $94(94.0 \%)$ & $6(6.0 \%)$ \\
\hline PMTCT & $153(40.0 \%)$ & $133(86.9 \%)$ & $20(13.1 \%)$ \\
\hline None & $129(33.8 \%)$ & $95(73.6 \%)$ & $34(26.4 \%)$ \\
\hline \multicolumn{4}{|l|}{ PMTCT regimen } \\
\hline Already on HAART & $100(26.2 \%)$ & $94(94.0 \%)$ & $6(6.0 \%)$ \\
\hline None & $129(33.8 \%)$ & $95(73.6 \%)$ & $34(26.4 \%)$ \\
\hline sdNVP & $91(23.8 \%)$ & $78(85.7 \%)$ & $13(14.3 \%)$ \\
\hline $\mathrm{sdNVP}+\mathrm{AZT}+3 \mathrm{TC}$ & $55(14.4 \%)$ & $48(87.3 \%)$ & $7(12.7 \%)$ \\
\hline sdNVP + AZT for 4 weeks & $7(1.8 \%)$ & $7(100.0 \%)$ & $0(0.0 \%)$ \\
\hline \multicolumn{4}{|l|}{ ARV prophylaxis at birth } \\
\hline Yes & $298(78.0 \%)$ & $276(92.6 \%)$ & $22(7.4 \%)$ \\
\hline No & $84(22.0 \%)$ & $46(54.8 \%)$ & $38(45.2 \%)$ \\
\hline \multicolumn{4}{|l|}{ Infant ARV regimen } \\
\hline None & $84(22.0 \%)$ & $46(54.8 \%)$ & $38(45.2 \%)$ \\
\hline sdNVP & $127(33.2 \%)$ & $114(89.8 \%)$ & $13(10.2 \%)$ \\
\hline sdNVP + AZT 4 wks & $36(9.4 \%)$ & $34(94.4 \%)$ & $2(5.6 \%)$ \\
\hline $\mathrm{AZT}+\mathrm{sdNVP}+3 \mathrm{TC}$ & $104(27.2 \%)$ & $98(94.2 \%)$ & $6(5.8 \%)$ \\
\hline Others & $31(8.1 \%)$ & $30(96.8 \%)$ & $1(3.2 \%)$ \\
\hline \multicolumn{4}{|l|}{ Feeding practice } \\
\hline ERF & $80(20.9 \%)$ & $78(97.5 \%)$ & $2(2.5 \%)$ \\
\hline $\mathrm{EBF}$ & $267(69.9 \%)$ & $233(87.3 \%)$ & $34(12.7 \%)$ \\
\hline $\mathrm{MF}$ & $35(9.2 \%)$ & $11(31.4 \%)$ & $24(68.6 \%)$ \\
\hline \multicolumn{4}{|l|}{ Growth pattern } \\
\hline Normal & $342(89.5 \%)$ & $291(85.1 \%)$ & $51(14.9 \%)$ \\
\hline Growth failure & $40(10.5 \%)$ & $31(77.5 \%)$ & $9(22.5 \%)$ \\
\hline
\end{tabular}

*AZT, zidovudine; sdNVP, single dose nevirapine; 3TC, lamivudine.

Of the total infants born to HIV positive mothers, 298 (78.0\%) took ARV prophylaxis at birth and 217 (56.8\%) received Cotrimoxazole prophylactic treatment. During the follow-up, 276 (69.9\%) infants were on exclusive breastfeeding while the $35(9.2 \%)$ were on mixed feeding. From the infants on care, 40 (10.5\%) of them had history of growth failure and two of them had red flag in the developmental staging.

At the start of exposed infant care, 379 (99.2\%) mothers were alive and $283(74.1 \%)$ were enrolled into care. Among the HIV positive mothers, $253(66.2 \%)$ of them were receiving
PMTCT intervention during pregnancy and at child birth, of which $100(26.2 \%)$ were already on ART. Almost all, 378 (99.0\%) mothers had normal breast condition during the follow-up (Table 1).

3.1. Rate of Maternal to Child HIV Transmission. During the follow-up, there was maternal to child HIV transmission in $60(15.7 \%)$ of HIV tested infants; most of them 55 (91.7\%) were confirmed by DNA-PCR. The transmission was higher in females than in males (17.6\% versus $13.8 \%)$. Fifty percent of infants delivered at home were HIV positive compared to 
TABLE 2: Determinants of mother-to-child transmission of HIV infection among exposed infants on follow-up care in Dire Dawa, Ethiopia, September 2014.

\begin{tabular}{|c|c|c|c|c|}
\hline Variables & COR (95\% CI) & $P$ value & $\operatorname{AOR}(95 \% \mathrm{CI})$ & $P$ value \\
\hline \multicolumn{5}{|l|}{ Residence } \\
\hline Urban & 1 & - & 1 & - \\
\hline Rural & $3.80(1.87,7.71)$ & 0.000 & $3.29(1.40,7.22)$ & 0.012 \\
\hline \multicolumn{5}{|l|}{ Place of delivery } \\
\hline Health institution & 1 & - & 1 & - \\
\hline Home & $7.39(3.62,15.10)$ & 0.000 & $3.35(1.58,8.38)$ & 0.010 \\
\hline \multicolumn{5}{|c|}{ ARV prophylaxis at birth } \\
\hline Yes & 1 & - & 1 & - \\
\hline No & $10.36(5.63,19.09)$ & 0.000 & $5.83(2.84,11.94)$ & 0.000 \\
\hline \multicolumn{5}{|l|}{ Feeding practice } \\
\hline ERF & 1 & - & 1 & - \\
\hline $\mathrm{EBF}$ & $5.69(1.34,24.24)$ & 0.019 & $4.86(1.11,21.40)$ & 0.036 \\
\hline $\mathrm{MF}$ & $85.10(17.62,410.87)$ & 0.000 & $42.21(8.31,214.38)$ & 0.000 \\
\hline \multicolumn{5}{|l|}{ Infant age at enrollment } \\
\hline$\leq 6$ weeks & 1 & - & 1 & - \\
\hline$>6$ weeks & $2.13(1.11,4.08)$ & 0.023 & $0.48(0.19,1.26)$ & 0.135 \\
\hline \multicolumn{5}{|l|}{ Mother enrolled } \\
\hline Yes & 1 & - & 1 & - \\
\hline No & $3.13(1.78,5.49)$ & 0.000 & $1.11(0.35,3.51)$ & 0.814 \\
\hline \multicolumn{5}{|l|}{ PMTCT intervention } \\
\hline On ART & 1 & - & 1 & - \\
\hline PMTCT & $2.36(0.91,6.09)$ & 0.077 & $1.76(0.60,5.11)$ & 0.282 \\
\hline None & $5.61(2.25,13.98)$ & 0.000 & $0.72(0.16,3.27)$ & 0.667 \\
\hline \multicolumn{5}{|c|}{ PMTCT mother-child pairs } \\
\hline Both on ARV & 1 & - & 1 & - \\
\hline Either one on ARV & $2.42(1.10,5.29)$ & 0.027 & $1.60(0.65,3.93)$ & 0.303 \\
\hline Neither on ARV & $9.21(4.69,18.08)$ & 0.000 & $4.42(2.01,9.82)$ & 0.000 \\
\hline
\end{tabular}

11.9\% among infants delivered at health institutions. The transmission rate was higher among infants who did not receive ARV prophylaxis at birth $(45.2 \%)$ compared to those who received $(7.4 \%)$ (Table 1$)$.

3.2. Determinants of Maternal to Child HIV Transmission. In the bivariate logistic regression analysis, mother's place of residence, infant's place of delivery, infant ARV prophylaxis at birth, feeding practice, infant age at enrollment, maternal enrollment into care, and PMTCT mother-child pairs were all associated with mother-to-child HIV transmission.

In the multivariate logistic regression analysis, being rural resident (AOR: 3.29; 95\% CI: 1.40, 7.22), delivery at home (AOR: 3.35; 95\% CI: 1.58, 8.38), infant not receiving ARV prophylaxis at birth (AOR: 5.83; 95\% CI: 2.84, 11.94), mixed feeding practice (AOR: 42.21; 95\% CI: 8.31, 214.38), and mother-child pairs not on PMTCT were found to be the most important significant determinants of mother-to-child HIV transmission (Table 2).

\section{Discussion}

In this institutional retrospective study of exposed infants, 60 became HIV positive during the follow-up. The determinant factors of mother-to-child HIV transmission were rural residency, delivery at home, infant not receiving ARV prophylaxis at birth, and mixed feeding.

The prevalence rate HIV infection among exposed infants in this study was $15.7 \%$, which was comparable to national prevalence rate of $17 \%$ [2] and to reports from similar studies in countries with resource-poor settings where the prevalence rate reported was $11 \%-21.8 \%[5,9,10]$. In spite of unrelenting global efforts made to eliminate MTCT and create a generation born HIV-free, the prevalence rate HIV infection in developing countries is still higher owing to low availability of PMTCT services for HIV positive pregnant women, elective caesarean sections, and avoidance of breastfeeding due to economic and cultural problem [1].

In this study, infants born to HIV positive mothers from rural residence were three times at higher risk (AOR: 3.29; $95 \%$ CI: $1.40,7.22$ ) of acquiring HIV infection than those born to mothers from urban areas. This finding falls in between figures reported from similar studies $[6,7]$. The observed risk difference could be attributable to limited number of ANC clinics providing PMTCT services in rural areas compared to urban ones coupled with better information and higher ANC attendance by pregnant mothers in urban areas [2]. Among 
mothers of HIV positive infants, $44 \%$ of mothers from rural areas had not received any ARV prophylaxis (ART or PMTCT) compared to $30 \%$ among mothers of urban residence.

Infants born at home had a threefold higher risk for HIV infection (AOR: 3.35; 95\% CI: 1.58, 8.38) compared to those delivered at health institutions. This is comparable to other studies from developing countries $[7,9,10]$. This could be because the risk of MTCT of HIV infection is minimized when attending skilled delivery in health institutions as it avails opportunities to ARV prophylaxes to the mother during labor and to the newborn right after birth. Regarding home deliveries in this study, $74 \%$ of pregnant mothers received PMTCT and only $16 \%$ of newborns received ARV prophylaxis at birth.

ARV prophylaxis at birth was another determinant factor for MTCT of HIV infection. Infants who did not receive ARV prophylaxis immediately after birth were 5.8 times at higher risk of being infected with HIV than their counterparts (AOR: 5.83; 95\% CI: 2.84, 11.94). This was similar to finding from other African countries [5].

Infants receiving mixed feeding were 42 times at higher risk of acquiring HIV infection compared to those receiving exclusive breastfeeding (AOR: 42.21; 95\% CI: 8.31, 214.38). A number of similar studies from resource-limited countries have also reported mixed feeding as an independent predictor of HIV transmission $[5,7,10,11]$. The possible justification for increased risk mixed fed infants would be that irritation of infant's immature gastrointestinal tract might facilitate entry of HIV viral particles within the mother's breast milk to the blood stream.

\section{Limitations of the Study}

This study has some limitations. Since the data was secondary, the information gathered was not complete for some infants or mothers. Not all maternal potential factors for vertical transmission were explored by the study. There were changes in PMTCT and HIV-exposed infant care protocols at different times but this research did not assess the difference with each protocol.

\section{Conclusion}

A higher prevalence of HIV infection was observed among exposed infants on follow-up at the HIV-exposed infant clinic. The risk of HIV transmission was higher among infants from rural areas, indicating scaling up of PMTCT program especially in rural settings. In addition, a higher risk of HIV infection among exposed infants delivered at home, infants who did not receive ARV after birth, and mixed fed infants suggests additional efforts by healthcare providers to intensify community mobilization and provide regular health education to HIV positive mothers, so as to increase PMTCT intervention, institutional delivery, and proper follow-up at the exposed infants care clinic. More prospective studies are needed to understand the influence of additional factors on MTCT, especially in rural settings.

\section{Conflict of Interests}

The authors declare that they have no competing interests.

\section{Authors' Contribution}

Fisseha Wudineh was the lead author who led the designing, data collection, analysis, and interpretation of results. Bereket Damtew participated in data collection, analysis, and inference of findings and narrated this paper for publication. All authors read and approved the final paper.

\section{Acknowledgments}

The authors would like to extend their gratitude and appreciation to the staff at Pediatric ART Clinic of Dil Chora Referral Hospital for their contributions in collection and quality control of data. They are also grateful to Dire Dawa Regional Health Bureau for its financial support and facilitation of conditions while carrying out this study.

\section{References}

[1] WHO UNAIDS, A Progress Report on the Global Plan towards the Elimination of New HIV Infections among Children by 2015 and Keeping Their Mothers Alive, WHO, UNAIDS, Geneva, Switzerland, 2012.

[2] FDRE-HAPCO, Country Progress Report on HIV/AIDS Response, Federal HIV/AIDS Prevention and Control Office, Addis Ababa, Ethiopia, 2012.

[3] HAPCO, Multi-Sectoral HIV/AIDS Response Annual Monitoring and Evaluation Report, July 2010-June 2011, Federal HIV/AIDS Prevention and Control Office, Addis Ababa, Ethiopia, 2011.

[4] FMOH, Accelerated Plan for Scaling Up Prevention of Mother to Child Transmission (PMTCT) Services in Ethiopia, Federal Ministry of Health, Addis Ababa, Ethiopia, 2011.

[5] F. Z. Gumbo, K. Duri, G. Q. Kandawasvika et al., "Risk factors of HIV vertical transmission in a cohort of women under a PMTCT program at three peri-urban clinics in a resource-poor setting," Journal of Perinatology, vol. 30, no. 11, pp. 717-723, 2010.

[6] M. Hussein, C. Jira, and B. Girma, "Assessment of effective coverage of HIV prevention of pregnant mother to child transmission services in Jimma Zone, South West Ethiopia," Ethiopian Journal of Health Sciences, vol. 21, no. 1, pp. 1-7, 2011.

[7] D. N. Koye and B. M. Zeleke, "Mother-to-child transmission of HIV and its predictors among HIV-exposed infants at a PMTCT clinic in northwest Ethiopia," BMC Public Health, vol. 13, article 398, 2013.

[8] A. H. Mirkuzie, S. G. Hinderaker, M. M. Sisay, K. M. Moland, and O. Mørkve, "Current status of medication adherence and infant follow up in the prevention of mother to child HIV transmission programme in Addis Ababa: a cohort study," Journal of the International AIDS Society, vol. 14, article 50, 2011.

[9] B. O. Ogunbosi, R. E. Oladokun, B. J. Brown, and K. I. Osinusi, "Prevalence and clinical pattern of paediatric HIV infection at the University College Hospital, Ibadan, Nigeria: a prospective cross-sectional study," Italian Journal of Pediatrics, vol. 37, no. 1, article 29, 2011.

[10] E. N. Kurewa, G. Q. Kandawasvika, F. Mhlanga et al., "Realities and challenges of a five year follow up of mother and child pairs 
on a PMTCT program in Zimbabwe," The Open AIDS Journal, vol. 5, no. 1, pp. 51-58, 2011.

[11] E. F. Ugochukwu and S. O. Kalu, "Early infant diagnosis of HIV infection in southeastern Nigeria: prevalence of HIV infection among HIV-exposed babies," West African Journal of Medicine, vol. 29, no. 1, pp. 3-7, 2010. 


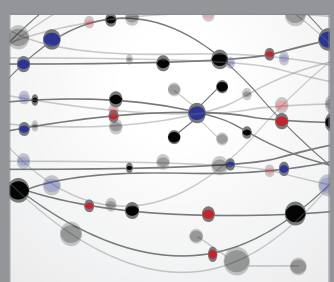

The Scientific World Journal
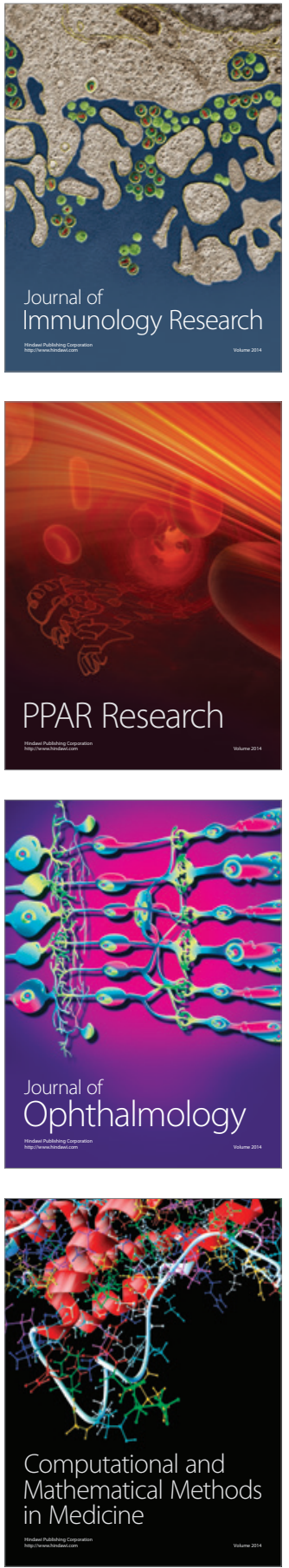

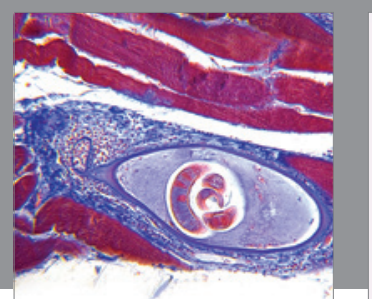

Gastroenterology Research and Practice

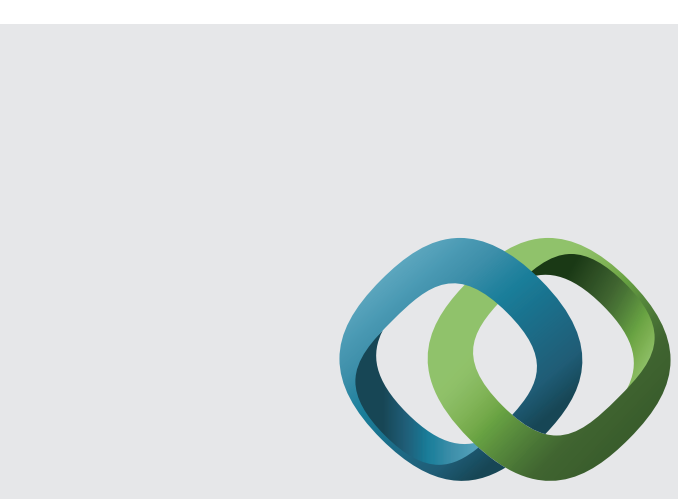

\section{Hindawi}

Submit your manuscripts at

http://www.hindawi.com
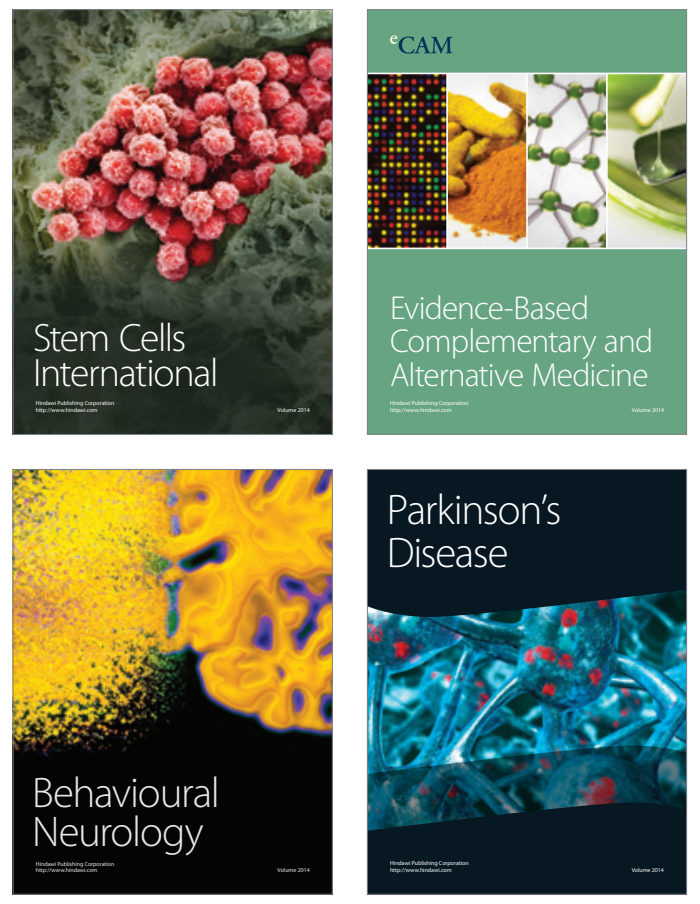
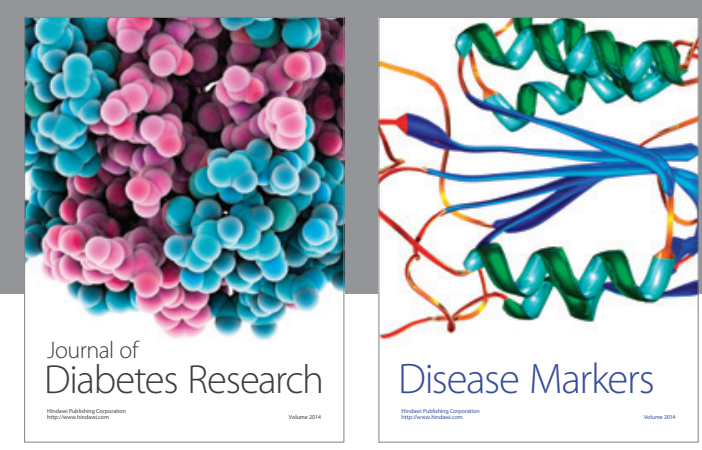

Disease Markers
